\title{
Oscillatory and Asymptotic Behavior of Solutions of Second Order Neutral Delay Difference Equations with "Maxima"
}

\author{
Ramalingam Arul, Manvel Angayarkanni \\ Department of Mathematics, Kandaswami Kandar's College, Namakkal, India \\ Email: drrarul@gmail.com, angayarkanni66@rediffmail.com
}

Received 14 January 2015; accepted 2 February 2015; published 6 February 2015

Copyright (C) 2015 by authors and Scientific Research Publishing Inc.

This work is licensed under the Creative Commons Attribution International License (CC BY). http://creativecommons.org/licenses/by/4.0/

\section{(c) (i) Open Access}

\section{Abstract}

In this paper, we study the oscillatory and asymptotic behavior of second order neutral delay difference equation with "maxima" of the form

$$
\Delta\left(a_{n} \Delta\left(x_{n}+p_{n} x_{n-\tau}\right)\right)+q_{n} \max _{[n-\sigma, n]} x_{s}^{\alpha}=0, \quad n \in \mathbb{N}\left(n_{0}\right)
$$

Examples are given to illustrate the main result.

\section{Keywords}

\section{Second Order, Oscillatory, Asymptotic Behavior, Neutral Delay Difference Equations with} "Maxima"

\section{Introduction}

Consider the oscillatory and asymptotic behavior of second order neutral delay difference equation with "maxima" of the form

$$
\Delta\left(a_{n} \Delta\left(x_{n}+p_{n} x_{n-\tau}\right)\right)+q_{n} \max _{[n-\sigma, n]} x_{s}^{\alpha}=0, \quad n \in \mathbb{N}\left(n_{0}\right),
$$

where $\Delta$ is the forward difference operator defined by $\Delta x_{n}=x_{n+1}-x_{n}$ and $\mathbb{N}\left(n_{0}\right)=\left\{n_{0}, n_{0}+1, n_{0}+2, \cdots\right\}$ and $n_{0}$ is a nonnegative integer subject to the following conditions:

$\left(C_{1}\right) \tau$ and $\sigma$ are positive integers;

$\left(C_{2}\right) \alpha$ is a ratio of odd positive integers;

$\left(C_{3}\right)\left\{p_{n}\right\}$ and $\left\{q_{n}\right\}$ are nonnegative real sequences with $\Delta p_{n} \geq 0$ and $0 \leq p_{n} \leq p<1$ for all $n \geq n_{0}$; 
$\left(C_{4}\right)\left\{a_{n}\right\}$ is a positive real sequence such that $\sum_{n=n_{0}}^{\infty} \frac{1}{a_{n}}<\infty$.

Let $\theta=\max \{\tau, \sigma\}$. By a solution of Equation (1), we mean a real sequence $\left\{x_{n}\right\}$ satisfying Equation (1) for all $n \geq n_{0}-\theta$. Such a solution is said to be oscillatory if it is neither eventually positive nor eventually negative and nonoscillatory otherwise.

From the review of literature it is well known that there is a lot of results available on the oscillatory and asymptotic behavior of solutions of neutral difference equations, see [1]-[5], and the references cited therein. But very few results are available in the literature dealing with the oscillatory and asymptotic behavior of solutions of neutral difference equations with "maxima", see [6]-[9], and the references cited therein. Therefore, in this paper, we investigate the oscillatory and asymptotic behavior of all solutions of Equation (1). The results obtained in this paper extend that in [4] for equation without "maxima".

In Section 2, we obtain some sufficient conditions for the oscillation of all solutions of Equation (1). In Section 3, we present some sufficient conditions for the existence of nonoscillatory solutions for the Equation (1) using contraction mapping principle. In Section 4, we present some examples to illustrate the main results.

\section{Oscillation Results}

In this section, we present some new sufficient conditions for the oscillation of all solutions of Equation (1). Throughout this section we use the following notation without further mention:

$$
\begin{gathered}
z_{n}=x_{n}+p_{n} x_{n-\tau}, \\
A_{n}=\sum_{s=n_{0}}^{n-1} \frac{1}{a_{s}}, \\
B_{n}=\sum_{s=n-\tau}^{\infty} \frac{1}{a_{s}},
\end{gathered}
$$

and

$$
C_{n}=a_{n} \sum_{s=n_{0}}^{n-1} \frac{1}{a_{s-\sigma}}
$$

Lemma 2.1. Let $\left\{x_{n}\right\}$ be an eventually positive solution of Equation (1). Then one of the following holds

(I) $z_{n}>0, \Delta z_{n}>0$ and $\Delta\left(a_{n} \Delta z_{n}\right) \leq 0$;

(II) $z_{n}>0, \Delta z_{n}<0$ and $\Delta\left(a_{n} \Delta z_{n}\right) \leq 0$.

Proof. Let $\left\{x_{n}\right\}$ be an eventually positive solution of Equation (1). Then we may assume that $x_{n-\sigma}>0$, $x_{n-\tau}>0$ for all $n \geq n_{0}$. Then inview of $\left(C_{3}\right)$ we have $z_{n}>0$ for all $n \geq n_{0}$. From the Equation (1), we obtain

$$
\Delta\left(a_{n} \Delta z_{n}\right)=-q_{n} \max _{[n-\sigma, n]} x_{s}^{\alpha} \leq 0 .
$$

Hence $a_{n} \Delta z_{n}$ and $z_{n}$ are of eventually of one sign. This completes the proof.

Lemma 2.2. Let $\left\{x_{n}\right\}$ be an eventually negative solution of Equation (1). Then one of the following holds

(I) $z_{n}<0, \Delta z_{n}<0$ and $\Delta\left(a_{n} \Delta z_{n}\right) \geq 0$;

(II) $z_{n}<0, \Delta z_{n}>0$ and $\Delta\left(a_{n} \Delta z_{n}\right) \geq 0$.

Proof. The proof is similar to that of Lemma 2.1.

Lemma 2.3. The sequence $\left\{x_{n}\right\}$ is an eventually negative solution of Equation (1) if and only if $\left\{-x_{n}\right\}$ is an eventually positive solution of the equation

$$
\Delta\left(a_{n} \Delta\left(x_{n}+p_{n} x_{n-\tau}\right)\right)+q_{n} \max _{[n-\sigma, n]} x_{s}^{\alpha}=0 .
$$

The assertion of Lemma 2.3 can be verified easily.

Lemma 2.4. Let $\left\{x_{n}\right\}$ be an eventually positive solution of Equation (1) and suppose Case (I) of Lemma 2.1 holds. Then there exists $N \in \mathbb{N}\left(n_{0}\right)$ such that

$$
\left(1-p_{n}\right) z_{n} \leq x_{n} \leq z_{n} \text { for all } n \geq N .
$$


Proof. From the definition of $z_{n}$ and condition $\left(C_{3}\right)$, we have $z_{n} \leq x_{n}$. Further $x_{n}=z_{n}-p_{n} x_{n-k} \geq z_{n}-p_{n} z_{n-k} \geq\left(1-p_{n}\right) z_{n}$, since $\left\{z_{n}\right\}$ is nondecreasing. This completes the proof.

Lemma 2.5. Let $\left\{x_{n}\right\}$ be an eventually positive solution of equation (1) and suppose Case (I) of Lemma 2.1 holds. Then there exists $N \in \mathbb{N}\left(n_{0}\right)$ such that

$$
z_{n-\sigma} \geq C_{n} \Delta z_{n} \text { for all } n \geq N .
$$

Proof. Since $\Delta\left(a_{n} \Delta z_{n}\right) \leq 0$, we see that

$$
z_{n-\sigma}=z_{N-\sigma}+\sum_{s=N}^{n-1} \Delta z_{s-\sigma} \geq a_{n-\sigma} \Delta z_{n-\sigma} \sum_{s=N}^{n-1} \frac{1}{a_{s-\sigma}}
$$

or

$$
z_{n-\sigma} \geq a_{n} \Delta z_{n} \sum_{s=N}^{n-1} \frac{1}{a_{s-\sigma}}
$$

The proof is now complete.

Lemma 2.6. Let $\left\{x_{n}\right\}$ be an eventually positive solution of Equation (1) and suppose Case (II) of Lemma 2.1 holds. Then there exists $N \in \mathbb{N}\left(n_{0}\right)$ such that $\left\{x_{n}\right\}$ is nonincreasing for all $n \geq N$.

Proof. Since $\Delta p_{n} \geq 0$ and $\Delta z_{n}=\Delta x_{n-k}+\Delta p_{n} x_{n-k}+p_{n+1} \Delta x_{n}<0$ then we have $\Delta x_{n} \leq 0$ for $n \geq N$. This completes the proof.

Theorem 2.1. Assume that $\alpha \geq 1$, and there exists a positive integer $k$ such that $\sigma \geq \tau-k$. If for all sufficiently large $n_{1} \in \mathbb{N}\left(n_{0}\right)$ and for all constants $M>0, L>0$. One has

$$
\sum_{n=n_{1}}^{\infty} A_{n+1} q_{n} \max _{[n-\sigma, n]}\left(1-p_{s}\right)^{\alpha}=\infty
$$

and

$$
\sum_{n=n_{1}}^{\infty}\left[B_{n+1}^{\alpha} q_{n} \max _{[n-\sigma, n]}\left(\frac{1}{1+p_{n-\tau}}\right)^{\alpha}-\frac{\alpha}{L^{\alpha-1} B_{n} a_{n-\tau}}\right]=\infty,
$$

then every solution of Equation (1) is oscillatory.

Proof. Assume to the contrary that there exists a nonoscillatory solution $\left\{x_{n}\right\}$ of Equation (1). Without loss of generality we may assume that $x_{n-\theta}>0$ for all $n \geq N \in \mathbb{N}\left(n_{0}\right)$, where $N$ is chosen so that both the cases of Lemma 2.1 hold for all $n \geq N$. We shall show that in each case we are led to a contradiction.

Case(I). From Lemma 2.4 and Equation (1), we have

$$
\Delta\left(a_{n}\left(\Delta z_{n}\right)\right)+q_{n} z_{n}^{\alpha} \max _{[n-\sigma, n]}\left(1-p_{s}\right)^{\alpha} \leq 0
$$

or

$$
\frac{\Delta\left(a_{n}\left(\Delta z_{n}\right)\right)}{z_{n}^{\alpha}} \leq-q_{n} \max _{[n-\sigma, n]}\left(1-p_{s}\right)^{\alpha} .
$$

Define $w_{n}=A_{n} \frac{a_{n} \Delta z_{n}}{z_{n}^{\alpha}}, n \geq N \geq n_{0}+k$, then we have

$$
\begin{aligned}
\Delta w_{n} & =A_{n+1} \frac{\Delta\left(a_{n} \Delta z_{n}\right)}{z_{n}^{\alpha}}+\frac{\Delta A_{n} a_{n} \Delta z_{n}}{z_{n}^{\alpha}}-\frac{A_{n+1} a_{n+1} \Delta z_{n+1}}{z_{n}^{\alpha} z_{n+1}^{\alpha}} \Delta z_{n}^{\alpha} \\
& \leq-A_{n+1} q_{n} \max _{[n-\sigma, n]}\left(1-p_{s}\right)^{\alpha}+\frac{\Delta A_{n} a_{n} \Delta z_{n}}{z_{n}^{\alpha}} \\
& \leq-A_{n+1} q_{n} \max _{[n-\sigma, n]}\left(1-p_{s}\right)^{\alpha}+\frac{\Delta z_{n}}{z_{n}^{\alpha}}
\end{aligned}
$$

or 


$$
\Delta w_{n} \leq-A_{n+1} q_{n} \max _{[n-\sigma, n]}\left(1-p_{s}\right)^{\alpha} .
$$

Summing the last inequality from $n_{1} \geq N$ to $n-1$, we have

$$
0<w_{n} \leq w_{n_{1}}-\sum_{s=n_{1}}^{n-1} A_{s+1} q_{s} \max _{[s-\sigma, s]}\left(1-p_{t}\right)^{\alpha} .
$$

Letting $n \rightarrow \infty$, we get a contradictions to (2).

Case(II). Define

$$
v_{n}=\frac{a_{n} \Delta z_{n}}{z_{n-\tau}^{\alpha}}, n \geq N
$$

Then $v_{n}<0$ for $n \geq N$. Since $\left\{a_{n} \Delta z_{n}\right\}$ is nonincreasing, we have

$$
\Delta z_{s} \leq \frac{a_{n} \Delta z_{n}}{a_{s}}, s \geq n
$$

Summing the last inequality from $n-\tau$ to $\ell-1$, we obtain

$$
z_{\ell} \leq z_{n-\tau}+a_{n} \Delta z_{n} \sum_{s=n-\tau}^{\ell-1} \frac{1}{a_{s}} .
$$

Since $z_{\ell}>0$ and $\Delta z_{\ell}<0$ by letting $\ell \rightarrow \infty$, in the last inequality we obtain

$$
0 \leq b \leq z_{n-\tau}+a_{n} \Delta z_{n} B_{n}, n \geq N
$$

or

$$
0 \leq z_{n-\tau}+a_{n} \Delta z_{n} B_{n}, n \geq N
$$

or

$$
B_{n} \frac{a_{n} \Delta z_{n}}{Z_{n-\tau}} \geq-1, n \geq N
$$

Thus

$$
-\frac{a_{n} \Delta z_{n}\left(-a_{n} \Delta z_{n}\right)^{-1}}{z_{n-\tau}^{\alpha}} B_{n}^{\alpha} \leq 1 .
$$

So, by $\Delta\left(-a_{n} \Delta z_{n}\right)>0$ and (6), we have

$$
-\frac{1}{L^{\alpha-1}} \leq v_{n} B_{n}^{\alpha} \leq 0, n \geq N,
$$

where $L=-a_{n} \Delta z_{N}$. From (6), we obtain

$$
\Delta v_{n}=\frac{\Delta\left(a_{n} \Delta z_{n}\right)}{z_{n-\tau}^{\alpha}}-\frac{a_{n+1} \Delta z_{n+1}}{z_{n-\tau}^{\alpha} z_{n+1-\tau}^{\alpha}} \Delta z_{n-\tau}^{\alpha} .
$$

By Mean Value Theorem,

$$
\Delta z_{n-\tau}^{\alpha}=z_{n+1-\tau}^{\alpha}-z_{n-\tau}^{\alpha}=\alpha t^{\alpha-1} \Delta z_{n-\tau}
$$

where $z_{n+1-\tau} \leq t \leq z_{n-\tau}$. Since $\alpha \geq 1$ and $\Delta z_{\tau(n)}<0$, we have

Therefore,

$$
\Delta z_{n-\tau}^{\alpha} \leq \alpha z_{n+1-\tau}^{\alpha-1} \Delta z_{n-\tau} .
$$

$$
\Delta v_{n} \leq-q_{n} \max _{[n-\sigma, n]} \frac{x_{s}^{\alpha}}{z_{s-\tau}^{\alpha}}-\frac{\alpha a_{n+1} \Delta z_{n+1} z_{n+1-\tau}^{\alpha-1} \Delta z_{n-\tau}}{z_{n-\tau}^{\alpha} z_{n+1-\tau}^{\alpha}} .
$$

Since $z_{n+1-\tau} \leq z_{n-\tau}$, we have 


$$
\Delta v_{n} \leq-q_{n} \max _{[n-\sigma, n]}\left(\frac{x_{s}}{z_{s-\tau}}\right)^{\alpha}-\frac{\alpha a_{n+1} \Delta z_{n+1}}{z_{n-\tau}^{\alpha+1}} \Delta z_{n-\tau} .
$$

From Lemma 2.6, $\Delta x_{n} \leq 0$ for $n \geq N$, we have

$$
\left(\frac{x_{n}}{z_{n-\tau}}\right)^{\alpha}=\left(\frac{x_{n}}{x_{n-\tau}+p_{n-\tau} x_{n-\tau-k}}\right)^{\alpha} \geq\left(\frac{1}{1+p_{n-\tau}}\right)^{\alpha} .
$$

From (8) and (9), we have

$$
\Delta v_{n}+q_{n} \max _{[n-\sigma, n]}\left(\frac{1}{1+p_{s-\tau}}\right)^{\alpha} \leq 0, n \geq N .
$$

Multiply (10) by $B_{n+1}^{\alpha}$ and summing it from $n_{1} \geq N$ to $n-1$, we have

$$
\sum_{s=n_{1}}^{n-1} B_{s+1}^{\alpha} \Delta v_{s}+\sum_{s=n_{1}}^{n-1} B_{s+1}^{\alpha} q_{s} \max _{[s-\sigma, s]}\left(\frac{1}{1+p_{t-\tau}}\right)^{\alpha} \leq 0 .
$$

Summation by parts formula yields

$$
\sum_{s=n_{1}}^{n-1} B_{s+1}^{\alpha} \Delta v_{s}=B_{n}^{\alpha} v_{n}-B_{n_{1}}^{\alpha} v_{n_{1}}-\sum_{s=n_{1}}^{n-1} v_{s} \Delta B_{s}^{\alpha} .
$$

Using Mean Value Theorem, we obtain

$$
\Delta B_{s}^{\alpha} \geq-\frac{\alpha B_{s}^{\alpha-1}}{a_{s-\tau}}
$$

Since $v_{n}<0$, we have

$$
\sum_{s=n_{1}}^{n-1} B_{s+1}^{\alpha} \Delta v_{s} \geq B_{n}^{\alpha} v_{n}-B_{n_{1}}^{\alpha} v_{n_{1}}-\sum_{s=n_{1}}^{n-1} \frac{\alpha v_{s} B_{s}^{\alpha-1}}{a_{s-\tau}}
$$

or

$$
B_{n}^{\alpha} v_{n}-B_{n_{1}}^{\alpha} v_{n_{1}}+\sum_{s=n_{1}}^{n-1} \frac{\alpha v_{s} B_{s}^{\alpha-1}}{a_{s-\tau}}+\sum_{s=n_{1}}^{n-1} B_{s+1}^{\alpha} q_{s} \max _{[s-\sigma, s]}\left(\frac{1}{1+p_{t-\tau}}\right)^{\alpha} \leq 0
$$

Therefore, from (7) and (11), we have

$$
B_{n}^{\alpha} v_{n} \leq B_{n_{1}}^{\alpha} v_{n_{1}}-\sum_{s=n_{1}}^{n-1}\left[B_{s+1}^{\alpha} q_{s} \max _{[s-\sigma, s]}\left(\frac{1}{1+p_{t-\tau}}\right)^{\alpha}-\frac{\alpha}{L^{\alpha-1} B_{s} a_{s-\tau}}\right] .
$$

Letting $n \rightarrow \infty$ in the last inequality, we obtain a contradiction to (3). This completes the proof.

Theorem 2.2. Assume that $\alpha \geq 1$, and there exists a positive integer $k$ such that $\sigma \geq \tau-k$. If for all sufficiently large $n_{1} \in \mathbb{N}\left(n_{0}\right)$ and for every constant $M>0$, (2) holds, and

$$
\sum_{n=n_{1}}^{\infty} B_{n+1}^{\alpha+1} q_{n} \max _{[n-\sigma, n]}\left(\frac{1}{1+p_{s-\tau}}\right)^{\alpha}=\infty,
$$

hold, then every solution of equation (1) is oscillatory.

Proof. Proceeding as in the proof of Theorem 2.1, we see that Lemma 2.1 holds for $n \geq N \in \mathbb{N}\left(n_{0}\right)$.

Case(I). Proceeding as in the proof of Theorem 2.1 (Case(I)) we obtain a contradiction to (12).

Case(II). Proceeding as in the proof of Theorem 2.1 (Case(II)) we obtain (7) and (10). Multiplying (10) by $B_{n+1}^{\alpha+1}$ and summing it from $n_{1} \geq N$ to $n-1$ we have

$$
\sum_{n=n_{1}}^{\infty} B_{s+1}^{\alpha+1} \Delta v_{s}+\sum_{s=n_{1}}^{n-1} B_{s+1}^{\alpha+1} q_{s} \max _{[s-\sigma, s]}\left(\frac{1}{1+p_{t-\tau}}\right)^{\alpha} \leq 0
$$


Using the summation by parts formula in the first term of the last inequality and rearranging, we obtain

$$
B_{n}^{\alpha+1} v_{n}-B_{n_{1}}^{\alpha+1} v_{n_{1}}+\sum_{s=n_{1}}^{n-1} \frac{(\alpha+1) v_{s} B_{s}^{\alpha}}{a_{s-\tau}}+\sum_{s=n_{1}}^{n-1} q_{s} B_{s}^{\alpha+1} \max _{[s-\sigma, s]}\left(\frac{1}{1+p_{t-\tau}}\right)^{\alpha} \leq 0
$$

Inview of (7), we have $-v_{n} B_{n}^{\alpha+1} \leq \frac{1}{L^{\alpha-1}} B_{n}<\infty$ as $n \rightarrow \infty$ and

$$
\sum_{s=n_{1}}^{n-1} q_{s} B_{s+1}^{\alpha+1} \max _{[s-\sigma, s]}\left(\frac{1}{1+p_{t-\tau}}\right)^{\alpha} \leq B_{n_{1}}^{\alpha+1} v_{n_{1}}-B_{n}^{\alpha+1} v_{n}+\frac{\alpha+1}{L^{\alpha-1}} \sum_{s=n_{1}}^{n-1} \frac{1}{a_{s-\tau}} .
$$

As $n \rightarrow \infty$ in the last inequality, we obtain a contradiction to (12). This completes the proof.

Theorem 2.3. Assume that $\alpha \geq 1$, and there exists a positive integer $k$ such that $\sigma \geq \tau-k$. If for all sufficiently large $n_{1} \in \mathbb{N}\left(n_{0}\right)$ and for every constant $M>0$, (2) holds, and

$$
\sum_{n=n_{1}}^{\infty} \frac{1}{a_{n}} \sum_{s=n_{1}}^{n-1} q_{s} B_{s}^{\alpha} \max _{[s-\sigma, s]}\left(\frac{1}{1+p_{t-\tau}}\right)^{\alpha}=\infty,
$$

then every solution of equation (1) is oscillatory.

Proof. Proceeding as in the proof of Theorem 2.1, we see that Lemma 2.1 holds and Case(I) is eliminated by the condition (2).

Case(II). Proceeding as in the proof of Theorem 2.1 (Case(II)) we have

$$
z_{\tau(n)} \geq-a_{n} \Delta z_{n} B_{n} \geq-a_{n_{1}} \Delta z_{n_{1}} B_{n}=c B_{n}
$$

where $c=-a_{n_{1}} \Delta z_{n_{1}}$. From Equation (1), we have

$$
\Delta\left(-a_{n} \Delta z_{n}\right)=q_{n} \max _{[n-\sigma, n]} x_{s}^{\alpha},
$$

and

$$
\frac{x_{n}}{Z_{n-\tau}} \geq \frac{1}{1+p_{n-\tau}}
$$

Hence

$$
\Delta\left(-a_{n} \Delta z_{n}\right) \geq c^{\alpha} q_{n} B_{n}^{\alpha} \max _{[n-\sigma, n]}\left(\frac{1}{1+p_{n-\tau}}\right)^{\alpha} .
$$

Summing the last inequality from $n_{1} \geq N$ to $n-1$, we obtain

$$
-a_{n} \Delta z_{n} \geq-a_{n_{1}} \Delta z_{n_{1}}+c^{\alpha} \sum_{s=n_{1}}^{n-1} q_{s} B_{s}^{\alpha} \max _{[s-\sigma, s]}\left(\frac{1}{1+p_{t-\tau}}\right)^{\alpha} \geq c^{\alpha} \sum_{s=n_{1}}^{n-1} q_{s} B_{s}^{\alpha} \max _{[s-\sigma, s]}\left(\frac{1}{1+p_{t-\tau}}\right)^{\alpha} .
$$

Again summing the last inequality from $n_{1} \geq N$ to $n-1$, we have

$$
z_{n_{1}} \geq z_{n_{1}}-z_{n} \geq c^{\alpha} \sum_{s=n_{1}}^{n-1} \frac{1}{a_{s}} \sum_{t=s_{1}}^{s-1} q_{t} B_{t}^{\alpha} \max _{[t-\sigma, t]}\left(\frac{1}{1+p_{i-\tau}}\right)^{\alpha} .
$$

Letting $n \rightarrow \infty$ in the above inequality, we obatin

$$
c^{\alpha} \sum_{s=n_{1}}^{n-1} \frac{1}{a_{s}} \sum_{t=s_{1}}^{s-1} q_{t} B_{t}^{\alpha} \max _{[t-\sigma, t]}\left(\frac{1}{1+p_{i-\tau}}\right)^{\alpha} \leq z_{n_{1}}
$$

a contradiction to (14). This completes the proof.

Next, we obtain sufficient conditions for the oscillation of all solutions of Equation (1) when $0<\alpha \leq 1$.

Theorem 2.4. Assume that $0<\alpha \leq 1$, and there exists a positive integer $k$ such that $\sigma \geq \tau-k$. If for all sufficiently large $n_{1} \in \mathbb{N}\left(n_{0}\right)$ and for every constant $M_{1}>0, L>0$, one has 


$$
\sum_{n=n_{1}}^{\infty}\left[A_{n+1} q_{n} \max _{[n-\sigma, n]}\left(1-p_{s}\right)^{\alpha}-\frac{\left(M_{1} A_{n}\right)^{1-\alpha}}{C_{n}}\right]=\infty
$$

and

$$
\sum_{n=n_{1}}^{\infty}\left[B_{n+1} q_{n} L^{1-\alpha} \max _{[n-\sigma, n]}\left(\frac{1}{1+p_{t-\tau}}\right)-\frac{1}{4 a_{n-\tau} B_{n+1}}\right]=\infty,
$$

then every solution of equation (1) is oscillatory.

Proof. Proceeding as in the proof of Theorem 2.1, we see that Lemma 2.4 holds for $n \geq N \in \mathbb{N}\left(n_{0}\right)$.

Case(I). Define $w_{n}$ by

$$
w_{n}=A_{n} \frac{a_{n} \Delta z_{n}}{z_{n}^{\alpha}}, n \geq N .
$$

Then $w_{n}>0$ and from Equation (1) and Lemma 2.2, we have

$$
\Delta w_{n} \leq-A_{n+1} q_{n} \max _{[n-\sigma, n]}\left(1-p_{s}\right)^{\alpha}+\frac{a_{n} \Delta z_{n}}{a_{n} z_{n}^{\alpha}} .
$$

Using Lemma 2.5 in (18), we obtain

$$
\Delta w_{n} \leq-A_{n+1} q_{n} \max _{[n-\sigma, n]}\left(1-p_{s}\right)^{\alpha}+\frac{z_{n}^{1-\alpha}}{C_{n}} .
$$

From the monotoncity of $\left\{a_{n} \Delta z_{n}\right\}$, we have

$$
z_{n}=z_{N}+\sum_{s=N}^{n-1} \frac{a_{s} \Delta z_{s}}{a_{s}} \leq z_{N}+a_{N} \Delta z_{N} R_{n} .
$$

and hence

$$
z_{n} \leq M_{1} R_{n}
$$

for some constant $M_{1}>0$ for all large $n$. Using (20) in (19) and then summing the resulting inequality from $n_{1} \geq N$ to $n-1$, we have

$$
0<w_{n} \leq w_{n_{1}}-\sum_{s=n_{1}}^{n-1}\left[A_{s+1} q_{s} \max _{[s-\sigma, s]}\left(1-p_{t}\right)^{\alpha}-\frac{\left(M_{1} A_{n}\right)^{1-\alpha}}{C_{n}}\right] .
$$

Letting $n \rightarrow \infty$ in (21), we obtain a contradiction to (16).

Case(II). Define a function $v_{n}$ by

$$
v_{n}=\frac{a_{n} \Delta z_{n}}{z_{n-\tau}}, n \geq N
$$

Then $v_{n}<0$ for $n \geq N$, we have

$$
\Delta v_{n}=\frac{\Delta\left(a_{n} \Delta z_{n}\right)}{z_{n-\tau}}-\frac{a_{n+1} \Delta z_{n+1}}{z_{n-\tau} z_{n+1-\tau}} \Delta z_{n-\tau} \leq-q_{n} \max _{[n-\sigma, n]}\left(\frac{x_{s}^{\alpha}}{z_{s-\tau}}\right)-\frac{a_{n} \Delta z_{n}}{z_{n-\tau} z_{n+1-\tau}} \Delta z_{n-\tau} .
$$

Since $\tau(n) \geq n$, and $a_{n} \Delta z_{n}$ is negative and decreasing we have

$$
a_{n-\tau} \Delta z_{n-\tau} \leq a_{n} \Delta z_{n} .
$$

Therefore

$$
\Delta v_{n} \leq-q_{n} \max _{[n-\sigma, n]}\left(\frac{x_{s}^{\alpha}}{z_{s-\tau}}\right)-\frac{\left(a_{n} \Delta z_{n}\right)^{2}}{a_{n-\tau} z_{n-\tau} z_{n+1-\tau}} .
$$

Since $z_{n}$ is a positive and decreasing, we have $z_{n+1-\tau} \leq z_{n-\tau}$. Combining the last two inequalities, we have 


$$
\Delta v_{n} \leq-q_{n} \max _{[n-\sigma, n]}\left(\frac{x_{s}^{\alpha}}{z_{s-\tau}}\right)-\frac{v_{n}^{2}}{a_{n-\tau}} .
$$

Now using (15) in (22), we obtain

$$
\Delta v_{n} \leq-q_{n} L^{\alpha-1} \max _{[n-\sigma, n]}\left(\frac{1}{1+p_{s-\tau}}\right)-\frac{v_{n}^{2}}{a_{n-\tau}}
$$

for some constant $L>0$. That is

$$
\Delta v_{n}+q_{n} L^{\alpha-1} \max _{[n-\sigma, n]}\left(\frac{1}{1+p_{s-\tau}}\right)+\frac{v_{n}^{2}}{a_{n-\tau}} \leq 0, n \geq N .
$$

Multiplying the last inequality by $B_{n+1}$, and then summing it from $n_{1} \geq N$ to $n-1$, we have

$$
\sum_{s=n_{1}}^{n-1} B_{s+1} \Delta v_{s}+\sum_{s=n_{1}}^{n-1} q_{s} L^{\alpha-1} B_{s+1} \max _{[s-\sigma, s]}\left(\frac{1}{1+p_{t-\tau}}\right)+\sum_{s=n_{1}}^{n-1} \frac{B_{s+1}}{a_{s-\tau}} v_{s}^{2} \leq 0 .
$$

Using the summation by parts formula in the first term of the above inequality and rearranging we obtain

$$
B_{n} v_{n}-B_{n_{1}} v_{n_{1}}+\sum_{s=n_{1}}^{n-1} q_{s} B_{s+1} L^{\alpha-1} \max _{[s-\sigma, s]}\left(\frac{1}{1+p_{t-\tau}}\right)+\sum_{s=n_{1}}^{n-1}\left(\frac{v_{s}}{a_{s-\tau}}+\frac{B_{s+1} v_{s}^{2}}{a_{s-\tau}}\right) \leq 0 .
$$

Using completing the square in the las term of the left hand side of the last inequality, we obtain

$$
B_{n} v_{n}-B_{n_{1}} v_{n_{1}}+\sum_{s=n_{1}}^{n-1} q_{s} B_{s+1} L^{\alpha-1} \max _{[s-\sigma, s]}\left(\frac{1}{1+p_{t-\tau}}\right)+\sum_{s=n_{1}}^{n-1} \frac{B_{s+1}}{a_{s-\tau}}\left(v_{s}+\frac{1}{2 B_{s+1}}\right)^{2}-\sum_{s=n_{1}}^{n-1} \frac{1}{4 a_{s-\tau} B_{s+1}} \leq 0
$$

or

$$
B_{n} v_{n} \leq B_{n_{1}} v_{n_{1}}-\sum_{s=n_{1}}^{n-1}\left[q_{s} B_{s+1} L^{\alpha-1} \max _{[s-\sigma, s]}\left(\frac{1}{1+p_{t-\tau}}\right)-\frac{1}{4 a_{s-\tau} B_{s+1}}\right] .
$$

Letting $n \rightarrow \infty$ in the above inequality, we obtain a contradiction to (17). The proof is now complete.

\section{Existence of Nonoscillatory Solutions}

In this section, we provide sufficient conditions for the existence of nonoscillatory solutions of Equation (1) in case $\alpha>1$ or $0<\alpha<1$. Note that in this section we do not require $p_{n} \equiv p$.

Theorem 3.1. Assume that $\alpha>1$. If

$$
\sum_{n=n_{0}}^{\infty} q_{n} A_{n-\sigma}^{\alpha}<\infty
$$

and

$$
\lim _{n \rightarrow \infty} \frac{A_{n-\tau}}{A_{n}}=1,
$$

then Equation (1) has a bounded nonoscillatory solution.

Proof. Choose $N \geq n_{0}$ sufficiently large so that

$$
p_{n} \frac{A_{n-\tau}}{A_{n}} \leq \frac{3 p+1}{4}
$$

and

$$
\sum_{s=N}^{\infty} q_{s} A_{s-\sigma}^{\alpha} \leq \frac{1-p}{8 \alpha}
$$

for $n \geq N$. Let $\chi$ be the set of all bounded real sequences defined for all $n \geq n_{0}$ with norm 


$$
\left\|x_{n}\right\|=\sup _{n \geq n_{0}}\left\{\frac{x_{n}}{A_{n}}\right\}
$$

and let

$$
S=\left\{x \in \chi ; \frac{3(1-p)}{8} \leq x_{n} \leq 1, n \geq n_{0}\right\} .
$$

Define a mapping $T: S \rightarrow \chi$ by

$$
(T x)_{n}= \begin{cases}\frac{3 p+5}{8} A_{n}-p_{n} x_{n-\tau}+A_{n} \sum_{s=N}^{n} q_{s} \max _{[s-\sigma, s]} x_{t}^{\alpha}+\sum_{s=n}^{\infty} q_{s} A_{s} \max _{[s-\sigma, s]} x_{t}^{\alpha}, & n \geq N \\ (T x)_{N}, & n_{0} \leq n<N .\end{cases}
$$

Clearly, $T$ is continuous. Now for every $x \in S$ and $n \geq N$, (25) implies

$$
\begin{aligned}
(T x)_{n} & \geq A_{n} \frac{3 p+5}{8}-p_{n} x_{n-\tau} \geq A_{n}\left(\frac{3 p+5}{8}-p_{n} \frac{A_{n-\tau}}{A_{n}}\right) \\
& \geq A_{n}\left(\frac{3 p+5}{8}-\frac{3 p+1}{4}\right) \geq \frac{3(1-p)}{8} A_{n} .
\end{aligned}
$$

Also, from (26) we have

$$
\begin{aligned}
(T x)_{n} & \leq A_{n} \frac{3 p+5}{8}+A_{n} \sum_{s=N}^{n} q_{s} \max _{[s-\sigma, s]} x_{t}^{\alpha}+\sum_{s=n}^{\infty} q_{s} A_{s} \max _{[s-\sigma, s]} x_{t}^{\alpha} \\
& \leq A_{n} \frac{3 p+5}{8}+A_{n} \sum_{s=N}^{\infty} q_{s} \max _{[s-\sigma, s]} A_{t}^{\alpha} \\
& \leq A_{n}\left(\frac{3 p+5}{8}+\frac{1-p}{8 \alpha}\right)<A_{n} .
\end{aligned}
$$

Thus, we have that $T S \subset S$. Since $S$ is bounded, closed and convex subset of $\chi$, we only need to show that $T$ is contraction mapping on $S$ in order to apply the contraction mapping principle. For $x, y \in S$ and $n \geq N$, we have

$$
\begin{aligned}
& \frac{1}{A_{n}}\left|(T x)_{n}-(T y)_{n}\right| \leq \frac{p_{n}}{A_{n}[n-\sigma, n]} \max _{s-\tau+\sigma}-y_{s-\tau+\sigma}\left|+\sum_{s=N}^{n} q_{s} \max _{[s-\sigma, s]}\right| x_{t}^{\alpha}-y_{t}^{\alpha}\left|+\frac{1}{A_{n}} \sum_{s=n}^{\infty} q_{s} A_{s} \max _{[s-\sigma, s]}\right| x_{t}^{\alpha}-y_{t}^{\alpha} \mid \\
& \leq p_{n} \frac{A_{n-\tau}}{A_{n}} \max _{[n-\sigma, n]}\left|\frac{x_{s-\tau}}{A_{s-\tau}}-\frac{y_{s-\tau}}{A_{s-\tau}}\right|+\sum_{s=N}^{\infty} q_{s} A_{s-\sigma}^{\alpha} \max _{[s-\sigma, s]}\left|\left(\frac{x_{t-\sigma}}{A_{t-\sigma}}\right)^{\alpha}-\left(\frac{y_{t-\sigma}}{A_{t-\sigma}}\right)^{\alpha}\right| .
\end{aligned}
$$

By the Mean Value Theorem applied to the function $f(u)=u^{\alpha}, \alpha>1$, we see that for any $x, y \in S$, we have $\left|x^{\alpha}-y^{\alpha}\right| \leq 2 \alpha|x-y|$ for all $n \geq N$. Hence

$$
\begin{aligned}
\|T x-T y\| & \leq \frac{3 p+1}{4}\|x-y\|+2 \alpha \sum_{s=N}^{\infty} q_{s} A_{s-\sigma}^{\alpha}\|x-y\| \\
& \leq \frac{3 p+1}{4}\|x-y\|+2 \alpha \frac{1-p}{8 \alpha}\|x-y\| \\
& =\frac{p+1}{2}\|x-y\|<\|x-y\| .
\end{aligned}
$$

Thus, $T$ is a contraction mapping, so $T$ has a unique fixed point $x \in S$ such that $T x=x$. It is easy to see that $x=\left\{x_{n}\right\}$ is a positive solution of Equation (1). This complete the proof of the theorem.

Theorem 3.2. Assume that $0<\alpha<1$. If

$$
\sum_{n=n_{0}}^{\infty} A_{n} q_{n}<\infty
$$


then Equation (1) has a bounded nonoscillatory solution.

Proof. Choose $N \geq n_{0}$ sufficiently large so that

$$
\sum_{s=N}^{\infty} q_{s} A_{s} \leq \frac{(1-p)^{2}}{8}
$$

Let $\chi$ be the set of all bounded real sequences defined for all $n \geq n_{0}$ with norm

$$
\|x\|=\sup _{n \geq n_{0}}\left\{x_{n}\right\}
$$

and let

$$
S=\left\{x \in \chi: \frac{3(1-p)}{8} \leq x_{n} \leq 1, n \geq n_{0}\right\} .
$$

Define a mapping $T: S \rightarrow \chi$ by

$$
(T x)_{n}=\left\{\begin{array}{lr}
\frac{3 p+5}{8}-p_{n} x_{n-\tau}+A_{n} \sum_{s=N}^{n} q_{s} \max _{[s-\sigma, s]} x_{t}^{\alpha}+\sum_{s=n}^{\infty} q_{s} A_{s} \max _{[s-\sigma, s]} x_{t}^{\alpha}, & n \geq N \\
(T x)_{N}, & n_{0} \leq n<N .
\end{array}\right.
$$

It is easy to see that $T$ is continuous, $T S \subset S$, and for any $x, y \in S$ and $n \geq N$, we have

$$
\left|(T x)_{n}-(T y)_{n}\right| \leq p_{n} \max _{[n-\sigma, n]}\left|x_{s-\tau}-y_{s-\tau}\right|+\sum_{s=N}^{\infty} q_{s} A_{s} \max _{[s-\sigma, s]}\left|x_{t}^{\alpha}-y_{t}^{\alpha}\right| .
$$

By the Mean Value Theorem applied to the function $f(u)=u^{\alpha}, 0<\alpha<1$, we see that for any $x, y \in S$, we have $\left|x^{\alpha}-y^{\alpha}\right| \leq \frac{8 \alpha}{3(1-p)}|x-y|$ for all $n \geq N$. Hence

$$
\|T x-T y\| \leq\|x-y\|\left(p+\frac{8 \alpha}{3(1-p)} \frac{(1-p)^{2}}{8}\right)<\|x-y\|,
$$

and we see that $T$ is a contraction on $S$. Hence, $T$ has a unique fixed point which is clearly a positive solution of Equation (1). This completes the proof of the theorem.

\section{Examples}

In this section we present some examples to illustrate the main results.

Example 4.1. Consider the difference equations

$$
\Delta\left(2^{n+1} \Delta\left(x_{n}+\frac{1}{3} x_{n-3}\right)\right)+2^{4 n} \max _{[n-1, n]} x_{s}^{3}=0, n \geq 0 .
$$

Here $\alpha=3, a_{n}=2^{n+1}, p_{n}=\frac{1}{3}, q_{n}=2^{4 n}$ and $\tau=3, \sigma=1$. Then $A_{n}=\frac{2^{n}-1}{2^{n}}, B_{n}=\frac{1}{2^{n-3}}$. Choosing $k=3$, we see that $\sigma \geq \tau-k$. Further it is easy to verify that all other conditions of Theorem 2.1 are satisfied. Therefore every solution of Equation (28) is oscillatory.

Example 4.2. Consider the difference equations

$$
\Delta\left(2^{n+1} \Delta\left(x_{n}+\frac{1}{4} x_{n-3}\right)\right)+2^{2 n} \max _{[n-2, n]} x_{s}^{\frac{1}{3}}=0, n \geq 0 .
$$

Here $\alpha=\frac{1}{3}, a_{n}=2^{n+1}, p_{n}=\frac{1}{4}, q_{n}=2^{4 n}$ and $\tau=3, \sigma=2$. Then $A_{n}=\frac{2^{n}-1}{2^{n}}, B_{n}=\frac{1}{2^{n-3}}$ and $C_{n}=2\left(2^{n}-1\right)$. Choosing $k=2$, we see that $\sigma \geq \tau-k$. Further it is easy to verify that all other conditions of Theorem 2.4 are satisfied. Therefore every solution of Equation (29) is oscillatory. 
Example 4.3. Consider the difference equations

$$
\Delta\left(n(n+1) \Delta\left(x_{n}+\frac{1}{4} x_{n-3}\right)\right)+n^{2} \max _{[n-1, n]} x_{s}^{3}=0, n \geq 1 .
$$

Here $\alpha=3, a_{n}=n(n+1), p_{n}=\frac{1}{4}, q_{n}=n^{2}$ and $\tau=3, \sigma=1$. By talking $A_{n}=\frac{1}{n}$, we see that all conditions of Theorem 3.1 are satisfied and hence Equation (30) has a bounded nonoscillatory solution.

Example 4.4. Consider the difference equations

$$
\Delta\left(n(n+1) \Delta\left(x_{n}+\frac{1}{2} x_{n-3}\right)\right)+\frac{1}{n[n-2, n]} x_{s}^{\frac{1}{3}}=0, n \geq 1 .
$$

Here $\alpha=\frac{1}{3}, a_{n}=n(n+1), p_{n}=\frac{1}{2}, q_{n}=\frac{1}{n}$ and $\tau=3, \sigma=2$. By talking $A_{n}=\frac{1}{n}$, we see that all conditions of Theorem 3.2 are satisfied and hence Equation (31) has a bounded nonoscillatory solution.

\section{References}

[1] Agarwal, R.P. (2000) Difference Equations and Inequalities. 2nd Edition, Marcel Dekker, New York.

[2] Agarwal, R.P., Bohner, M., Grace, S.R. and O’Regan, D. (2005) Discrete Oscillation Theory. Hindawi Publ. Corp., New York. http://dx.doi.org/10.1155/9789775945198

[3] Kelley, W.G. and Peterson, A.C. (2001) Difference Equations: An Introduction with Applications. 2nd Edition, Academic Press, New York.

[4] Thandapani, E. and Selvarangam, S. (2012) Oscillation of Second Emden-Fowler Type Neutral Difference Equations. Dynamics Continous Discrete Impulise System, 19, 453-469.

[5] Zhang, G. and Geo, Y. (2001) Oscillation Theory for Difference Equations. Publishing House of Higher Education, Beijing.

[6] Arul, R. and Angayarkanni, M. (2013) Asymptotic Behavior of Second Order Nonlinear Neutral Difference Equations with "Maxima". Far East Journal of Mathematical Science, 82, 79-92.

[7] Arul, R. and Angayarkanni, M. (2014) Oscillatory and Asymptotic Behavior of Second Order Neutral Difference Equations with "Maxima". Journal of Advances in Mathematics, 1916-1924.

[8] Luo, J.W. and Bainov, D.D. (2001) Oscillatory and Asymptotic Behavior of Second-Order Neutral Difference Equations with "Maxima”. Journal of Computational and Applied Mathematics, 131, 333-341. http://dx.doi.org/10.1016/S0377-0427(00)00264-8

[9] Luo, J.W. and Petrov, V.A. (1999) Oscillation of Second Order Neutral Difference Equations with "Maxima”. Journal of Mathematical Sciences Research. Hot-Line, 3, 17-22. 
Scientific Research Publishing (SCIRP) is one of the largest Open Access journal publishers. It is currently publishing more than 200 open access, online, peer-reviewed journals covering a wide range of academic disciplines. SCIRP serves the worldwide academic communities and contributes to the progress and application of science with its publication.

Other selected journals from SCIRP are listed as below. Submit your manuscript to us via either submit@scirp.org or Online Submission Portal.
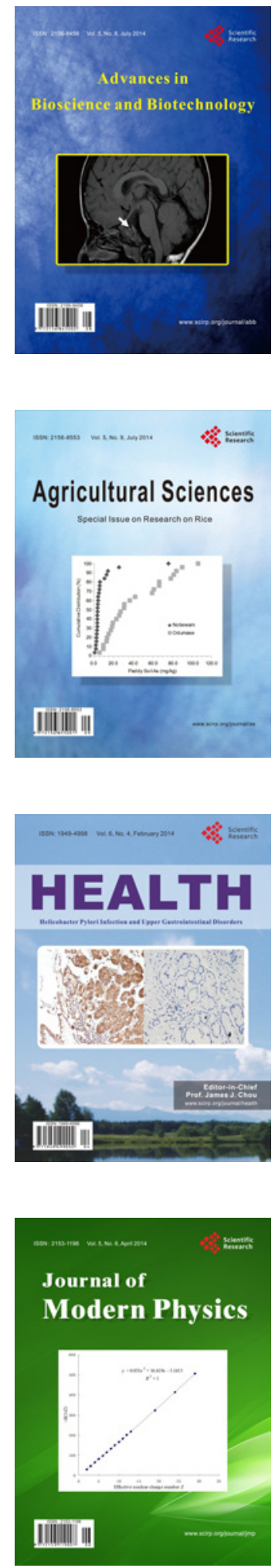
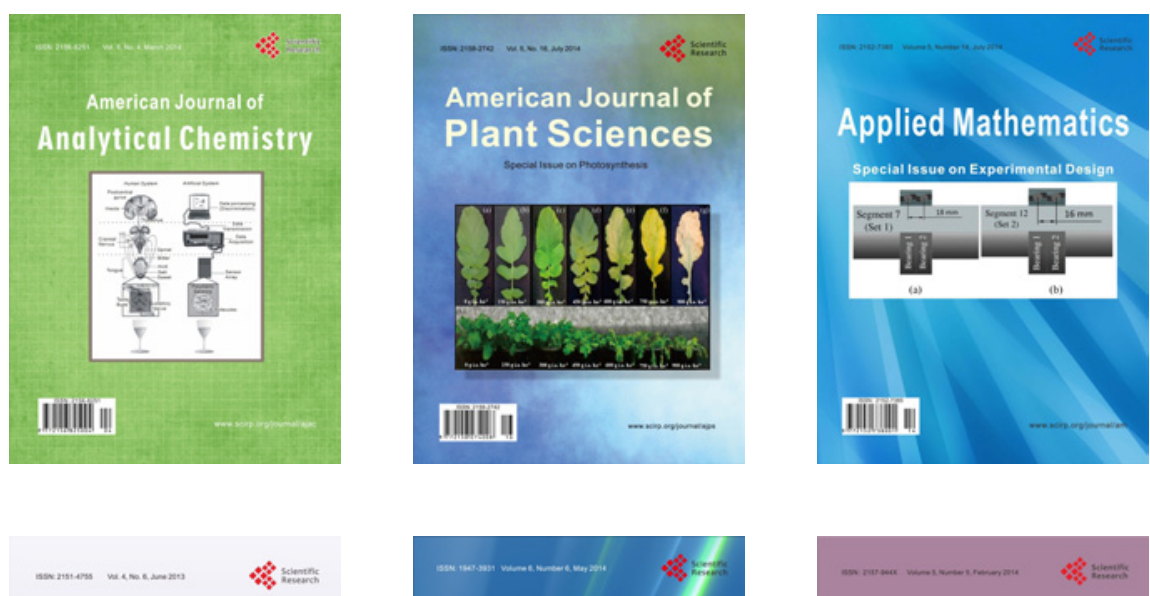

Creative Education
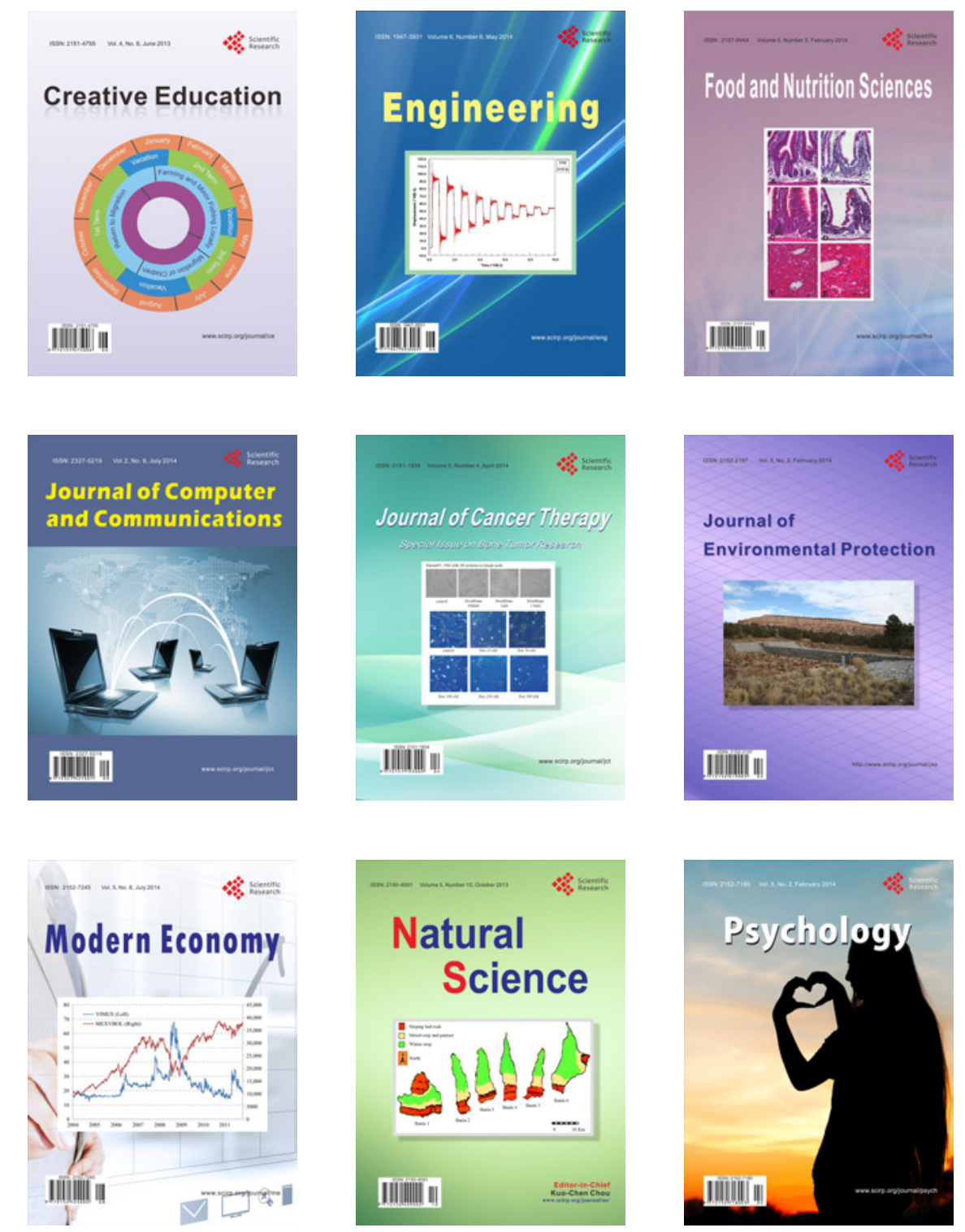